\title{
Domain Walls in Bent Nanowires
}

\author{
F. Lofink, ${ }^{1, *}$ A. Philippi-Kobs, ${ }^{1,2, \dagger}$ M. R. Rahbar Azad, ${ }^{1}$ S. Hankemeier, ${ }^{1}$ G. Hoffmann, ${ }^{1,}$ R. Frömter, ${ }^{1}$ and H. P. Oepen ${ }^{1,2}$ \\ ${ }^{1}$ Institut für Nanostruktur- und Festkörperphysik, Universität Hamburg, \\ Jungiusstraße 11, 20355 Hamburg, Germany \\ ${ }^{2}$ The Hamburg Centre of Ultrafast Imaging, Luruper Chaussee 149, 22761 Hamburg, Germany \\ (Received 21 November 2016; revised manuscript received 3 June 2017; published 17 August 2017)
}

\begin{abstract}
The influence of geometric parameters on the magnetic fine structure of domain walls in bent nanowires is investigated. The domain pattern in the soft-magnetic $\mathrm{Co}_{39} \mathrm{Fe}_{54} \mathrm{Si}_{7}$ alloy is studied via scanning electron microscopy with polarization analysis and modeled via micromagnetic simulations. It is demonstrated that the bending angle affects details of the microstructure as well as the preponderant domain-wall type. A phenomenological model is developed that provides the global energy minimum of individual types of domain walls as a function of the geometric parameters of the wire. The results can be directly transferred to permalloy wires, as permalloy and $\mathrm{Co}_{39} \mathrm{Fe}_{54} \mathrm{Si}_{7}$ alloy have a comparable magnetostatic exchange length.
\end{abstract}

DOI: $10.1103 /$ PhysRevApplied.8.024008

\section{INTRODUCTION}

Soft-magnetic nanowires containing two or more magnetic domains are in the very focus of recent research [1-5]. Describing and controlling the properties of the magnetic fine structure between two domains in a wire, which is commonly addressed as a domain wall, is a field of intense work [6-12]. Promising technological applications, like the magnetic racetrack memory [13], new sensor devices [14], and logic concepts for magnetic computing [15] have been proposed and are now under development.

Three distinct types of domain walls exist in softmagnetic wires with a head-to-head or tail-to-tail orientation of domain magnetization, i.e., the vortex wall, (symmetric) transverse wall, and asymmetric transverse wall [16-19]. The microstructure of the vortex wall consists of an in-plane magnetization that curls around a central, sharp quasisingularity, the vortex core. In the core, the magnetization points out of plane. In the transverse wall, the magnetization rotates within the film plane, creating a magnetization orientation that is transverse to the wire direction with poles at the wire edges. The microstructure that is formed can be either symmetric or asymmetric with respect to the wall center.

The type of domain wall depends on the dimensions of the wire (width and thickness), on the magnetic properties of the wire material, and on the magnetic history reflecting either a local or the global energy minimum [7]. Recently, phase diagrams for domain walls in straight wires and

\footnotetext{
*Present address: Fraunhofer Institute for Silicon Technology, Fraunhoferstr. 1, 25524 Itzehoe, Germany. fabian.lofink@isit.fraunhofer.de

${ }^{\dagger}$ Present address: Deutsches Elektronen-Synchrotron (DESY), Notkestraße 85, 22607 Hamburg, Germany.

${ }^{\star}$ Present address: Department of Physics, National Tsing Hua University, Hsinchu, Taiwan.
}

nanorings have been published $[8,16,17,20]$. Qualitatively, the transverse domain wall is the global minimum for thin and narrow wires. In contrast, the vortex domain wall is the predominant micromagnetic state in thick and wide wires. In the parameter range between these two types of microstructures, an asymmetric transverse domain wall has been identified as the third stable magnetic microstructure in straight wires [17]. The asymmetric transverse domain wall was experimentally first observed in 2007 [21], while a systematic investigation of the parameters that stabilize the asymmetric wall as well as an experimental proof of the predicted stability is still missing. For rather wide and thick wires, simulations predict that complex patterns exhibiting several vortices (and antivortices) form the domain wall [8].

A common strategy to nucleate domain walls within wires is to introduce a geometrical feature which acts as a pinning site for the domain wall. Typical pinning sites are notches or antinotches in straight or curved wires $[20,22,23]$. Alternatively, zigzag wires are used [24], in which domain walls can be easily created via an in-plane seeding field that is applied perpendicular to the main wire direction [25]. In spite of the frequent use of bent wires [24,26-36], the influence of the wire geometry on the magnetic fine structure of the domain wall has not been investigated yet. Knowing the exact magnetic fine structure, however, is necessary for a precise analysis and interpretation of the corresponding experimental results.

In general, all the above-described microstructures will show up in soft-magnetic wires, while variations of the relevant magnetic parameters will cause shifts of the absolute scale of phase boundaries. Nevertheless, almost all of the studies on the microstructure in wires have been performed utilizing permalloy as the prototype material.

The topic of this paper is the influence of the geometric parameters wire width $w$, wire thickness $t$, and bending angle $\alpha$ on the prevalent type of domain wall and its fine 
structure in V-shaped nanowires (i.e., zigzag wires with only one bend). The experimental study is performed by means of scanning electron microscopy with polarization analysis (SEMPA) [37-39]. As the spin polarization of the secondary electrons, which is crucial for SEMPA contrast [40], is low for permalloy, we choose a different softmagnetic material, $\mathrm{Co}_{39} \mathrm{Fe}_{54} \mathrm{Si}_{7}$, which gives a high spin polarization of the secondaries. From the micromagnetic point of view, $\mathrm{Co}_{39} \mathrm{Fe}_{54} \mathrm{Si}_{7}$ is very similar to permalloy, as the materials have almost the same magnetostatic exchange length. Hence, we keep the connection to the majority of studies that have so far been published on the microstructure in soft-magnetic wires. The experimental results are compared to micromagnetic simulations, which are performed using OOMMF [41]. Based on the main features of the domain-wall types a phenomenological model is developed that particularly provides the prevalent type of domain wall in dependence of the geometric and magnetic parameters of the wire.

\section{EXPERIMENTAL DETAILS}

\section{A. Preparation of $\mathbf{V}$-shaped soft-magnetic wires}

Magnetic wires containing one kink ( $\mathrm{V}$-shaped) are fabricated with different geometric parameters (Fig. 1). The wire width $w$ is varied from 100 to $400 \mathrm{~nm}$ (400 to $800 \mathrm{~nm})$ in steps of $50 \mathrm{~nm}(200 \mathrm{~nm})$, respectively [42], while the bending angle is kept constant at $\alpha=150^{\circ}$ [see Figs. 1(b) -1 (d); $\alpha=180^{\circ}$ corresponds to a straight wire]. In a second set, the bending angle is changed from $20^{\circ}$ to $180^{\circ}$ in increments of $10^{\circ}$ [see Figs. $1(\mathrm{e})-1(\mathrm{~g})$ ] at a constant width of $400 \mathrm{~nm}$. Each set of wires is structured out of soft-magnetic films with three different thicknesses $t=10,20$, and $30 \mathrm{~nm}$ via focused-ion-beam (FIB) milling using $\mathrm{Ga}^{+}$ions of

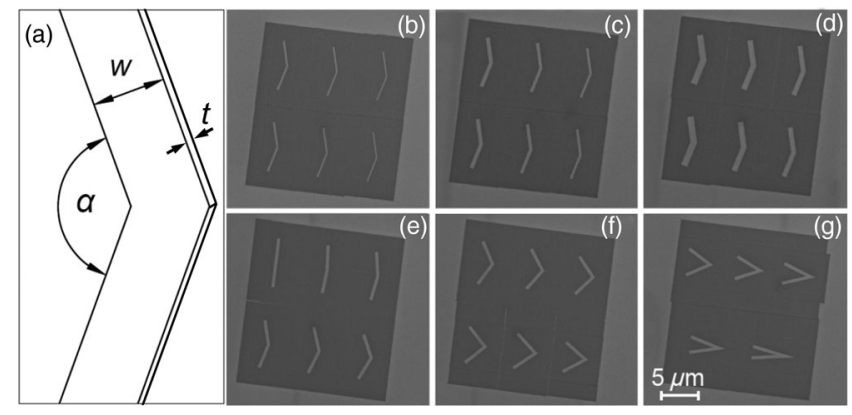

FIG. 1. (a) Sketch of a nanowire and definition of the geometric parameters. In (b)-(g), SEM micrographs of wire arrays with varied geometries are shown. Bright areas indicate magnetic material. Each array consists of six wires, which are carved out of the magnetic film by FIB milling. The area where the film material is removed appears dark. Outside the dark region, the pristine magnetic film can be recognized. In (b)-(d) the width while in $(\mathrm{e})-(\mathrm{g})$ the angle is varied. Each $(\alpha, w)$ set is realized in three different film thicknesses $(10,20$, and $30 \mathrm{~nm})$.
$30 \mathrm{keV}$ [43]. SEM micrographs are used to check for the quality of structuring and controlling the geometries.

\section{B. Properties of $\mathrm{Co}_{39} \mathrm{Fe}_{54} \mathrm{Si}_{7}$ thin films}

$\mathrm{Co}_{39} \mathrm{Fe}_{54} \mathrm{Si}_{7}$ is chosen for this study, as the secondary electrons from $\mathrm{Fe}$ and $\mathrm{Co}$ (in contrast to $\mathrm{Ni}$ ) are highly spin polarized [37], which is most advantageous for the SEMPA investigation as the magnetic image contrast scales with the spin polarization (asymmetry 5.6\%) [40]. In contrast, the asymmetry of permalloy $\left(\mathrm{Ni}_{80} \mathrm{Fe}_{20}\right)$ frequently used as a soft-magnetic material is only $2.3 \%$ due to the high amount of Ni.

The magnetic films are grown via electron-beam evaporation utilizing a target with the composition Co $0.3 \mathrm{~g}, \mathrm{Fe} 0.12 \mathrm{~g}$, and $\mathrm{Si} 0.12 \mathrm{~g}$ (rate $=0.5 \AA / \mathrm{s}$, base pressure $=2 \times 10^{-8} \mathrm{mbar}$ ). The material flux is controlled via a thickness monitor (quartz-crystal oscillator). The accuracy of the thickness is cross-checked by height profiling utilizing atomic force microscopy. Naturally oxidized $\mathrm{Si}(100)$ wafers are used as substrates. The composition of the deposited magnetic films is $\mathrm{Co}_{39} \mathrm{Fe}_{54} \mathrm{Si}_{7}$ (at. \%) which is determined by energy-dispersive $\mathrm{x}$-ray spectroscopy using the target as the calibration standard. The magnetic characterization is performed via SEMPA, longitudinal magneto-optical Kerr effect (MOKE) [44,45], and ferromagnetic resonance (FMR) [46].

Figure 2(a) displays the hysteresis curves obtained by MOKE for a magnetic field applied along two perpendicular in-plane directions. The direction of highest remanence (easy axis) is indicated by an angle of $0^{\circ}$. The decrease in remanence at $90^{\circ}$ (hard axis) is only $7 \%$. This result demonstrates that the system exhibits a very weak uniaxial anisotropy averaged over the spot size of the laser beam $(1 \mathrm{~mm})$. No magnetic field is intentionally applied during film growth. The weak anisotropy is most likely due to a small residual field that is effective during growth. As the magnetization reversal is dominated by domain nucleation and domain-wall movement, the MOKE measurements cannot be used to extract anisotropy constants. Because of the fact that the magnetization behavior is nearly isotropic, we conclude that the magnetocrystalline anisotropy is negligibly small compared to the magnetostatic energy that comes along with confining the size [16]. Hence, in a good approximation the micromagnetic configuration of $\mathrm{Co}_{39} \mathrm{Fe}_{54} \mathrm{Si}_{7}$ is governed by magnetostatic and exchange energy.

The saturation magnetization of the film is $\mu_{0} M_{S}=1.8 \mathrm{~T}$, which is determined via FMR measurements. The value for the exchange stiffness $A=35 \times 10^{-12} \mathrm{~J} / \mathrm{m}$ is taken from Ref. [47], which is a good approximation for the CoFeSi alloy as reasoned in Ref. [48]. For a straight wire, the micromagnetic structure is depending on film thickness $t$, width of the wire $w$, and the magnetostatic exchange length, defined as $\delta=\sqrt{2 A /\left(\mu_{0} M_{S}^{2}\right)}$ [16]. For $\mathrm{Co}_{39} \mathrm{Fe}_{54} \mathrm{Si}_{7}$, we obtain an exchange length of $3.7 \mathrm{~nm}$. The value is very 

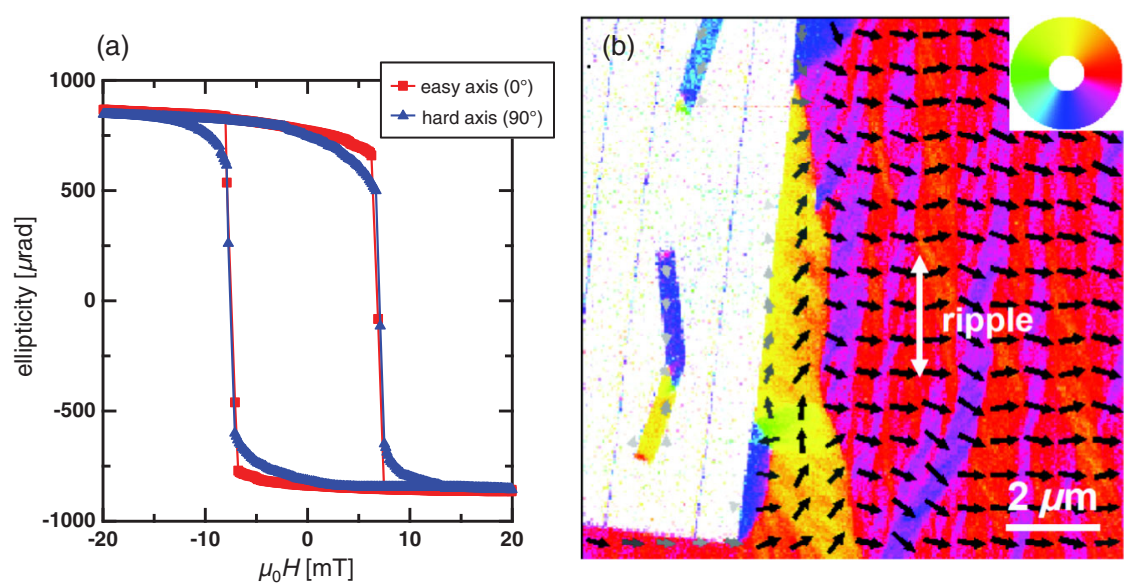

FIG. 2. (a) Magnetization behavior of the unstructured film with a thickness of $20 \mathrm{~nm}$ for a magnetic field applied along two perpendicular in-plane directions with the highest and lowest remanence. The curves are obtained utilizing the longitudinal magneto-optical Kerr effect. (b) SEMPA image of a 20-nm-thick nanowire and the adjacent magnetic film. The magnetization orientation is color coded and indicated by arrows. In the bright area, which is the area where the magnetic film is removed, no magnetic signal is obtained. close to the exchange length of permalloy, which is $4 \mathrm{~nm}$ $\left(A=1.3 \times 10^{-11} \mathrm{~J} / \mathrm{m}\right.$ and $M_{S}=8.0 \times 10^{5} \mathrm{~A} / \mathrm{m}$ [16]). Hence, the energy landscape of the magnetic microstructure in an object of limited size should be very similar for both soft-magnetic materials. Hence, the results of the present study can be transferred almost one by one (within the difference given by the deviation of the exchange lengths) to wires made from permalloy.

Figure 2(b) is a SEMPA micrograph showing the magnetic fine structure of nanowires and the remaining film prior to any field treatment. In the vicinity of the milled region (removed film), the magnetization is aligned parallel to the edges. Within the magnetic film, a ripple domain structure is visible, which is well known for polycrystalline permalloy films [52-54]. The characteristic pattern of rhombic-elongated structures comes from the interplay of uniaxial film anisotropy and exchange interaction on the one hand and local variations of cubic anisotropy on the other hand. The latter indicates that the film consists of randomly oriented grains. The grain size of the films is determined by scanning electron microscopy to be about $10 \mathrm{~nm}$. The microscopic variations in the anisotropy cancel out on a macroscopic scale and cannot be identified in the magnetization curves [Fig. 2(a)].

\section{EXPERIMENTAL PROCEDURE}

SEMPA is used for the simultaneous mapping of two orthogonal in-plane magnetization components $[37,40]$ at the surface. Magnetization images with a lateral resolution of $20 \mathrm{~nm}$ and a high orientation sensitivity $<4^{\circ}$ are acquired using a primary beam of $6 \mathrm{nA}$ at $8 \mathrm{keV}$. The SEMPA instrument operates in ultrahigh vacuum (base pressure of $5 \times 10^{-11} \mathrm{mbar}$ ). The samples are transferred from ambient conditions into the microscope. The surface contamination is removed via $\mathrm{Ar}^{+}$-ion milling at $600 \mathrm{eV}$. After sputter cleaning, the magnetic contrast is stable for hours.

The SEMPA micrograph in Fig. 3(a) shows the magnetic structure of six wires as fabricated, i.e., before the application of magnetic fields. The black-white contrast gives the magnetization component along the vertical image direction. The wires are predominantly $(75 \%)$ in a multidomain state containing one or more domain walls. A straightforward explanation of the multidomain configuration as the virgin state is based on the locally varying orientation of crystallites and thus principal axes of the magnetocrystalline anisotropy. During FIB fabrication, edges are created, and, hence, a shape anisotropy is generated and increases gradually. At some point during fabrication, the averaged magnetocrystalline anisotropy is compensated by the magnetostatic (shape) anisotropy energy and the varying local anisotropy gets an impact on the local orientation of magnetization. Thus, the tilting of magnetization along the wire axes can be in opposite directions throughout the wire, and multidomain states evolve that are stable due to pinning of the domain walls.

The focus of the present study is on the domain-wall type and fine structure of the walls pinned at the bend of the nanowires. To create a domain wall at this position, we apply an external magnetic field $\left(\left|B_{\text {ext }}\right| \approx 60 \mathrm{mT}\right)$ parallel (or antiparallel) to the direction indicated by the arrow in Fig. 3(b), which is inclined by $10^{\circ}$ with respect to the symmetry axis of the wires. The accuracy of the field direction is $\pm 5^{\circ}$. While the field is applied, the

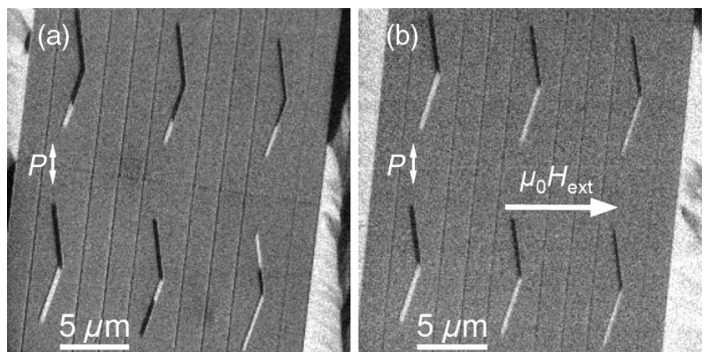

FIG. 3. SEMPA micrographs obtained with one polarizationsensitive axis of the polarization analyzer. The polarizationsensitive axis (indicated by $P$ ) is along the vertical of the SEM micrographs. (a) displays the magnetic structure in the "as-fabricated" state, while the micrograph in (b) gives the domain pattern after applying an external field of $B_{\text {ext }} \approx 60 \mathrm{mT}$ along the direction indicated by the white arrow. 
magnetization within the wires is tilted towards the direction of the external field. The field strength is sufficient to delete the former microstate, even if for small $w$ and large $\alpha$ the initial state is not a single domain [25]. After switching off the field, the magnetization relaxes into a two-domain state with the domain wall localized at the bend [Fig. 3(b)]. A "head-to-head" or "tail-to-tail" configuration is generated with the orientation of magnetization in the domains parallel to the long axes of the arms. The success rate to introduce a domain wall at the bend is about $95 \%$ for bending angles $\alpha \leq 160^{\circ}$. For larger bending angles (almost straight wires), single-domain states are predominantly found as expected. Each array of wire configurations $(\alpha, w, t)$ is studied at least two times to verify the dominant wall type [see Fig. 9(a)]. Identical arrays were studied after a new magnetization reversal was made.

\section{RESULTS AND DISCUSSION}

In the following, we discuss the magnetic fine structure of nanowires and compare the experimental results with micromagnetic simulations utilizing OOMMF [41]. The aforementioned magnetic parameters of $\mathrm{Co}_{39} \mathrm{Fe}_{54} \mathrm{Si}_{7}$ are taken. As the cell size in the simulations, a cube of $4 \mathrm{~nm} \times 4 \mathrm{~nm} \times$ wire height $/ 3$ is used. To numerically calculate the energy of the different wall types in dependence on the geometry of the wire, a magnetic structure that mimics the main features of the corresponding final state (energy minimum) is taken as the initial state. A damping constant of $\alpha_{\text {damping }}=0.5$ is used for the relaxation process, and the energy minimization is terminated when the net field that creates a torque is smaller than $1 \times 10^{-2} \mathrm{~A} / \mathrm{m}$. To extract the domain-wall energy differences between the transverse wall and vortex wall, it is ensured that the magnetization outside the wall area is equal in both cases, especially the orientation of the closure domains at the ends of the wire.

This section is divided into four main subsections: First, we discuss in Sec. IVA the different types of domain walls in a bent nanowire and their magnetic fine structure. Sections IV B and IV C deal with the impact of varying the geometrical parameters [thickness and wire width (Sec. IV B) and bending angle (Sec. IV C), respectively] on the magnetic structure and wall type. Based on the experimental data, a three-dimensional phase diagram is created where the preponderant domain-wall type is given as a function of the three geometrical parameters. The experimental results are compared with micromagnetic simulations. In Sec. IV D, a phenomenological model is given that describes the transition from vortex to transverse walls in this diagram.

\section{A. Domain-wall types and their magnetic fine structure}

In Fig. 4, SEMPA images of the three generic types of domain walls that predominantly exist for soft-magnetic wires with head-to-head and tail-to-tail domain orientation are shown, i.e., the transverse wall (Fig. 4 left), the vortex wall (Fig. 4 center), and the asymmetric transverse wall (Fig. 4 right). All three types of domain wall are similar to the corresponding wall structures in a straight wire segment
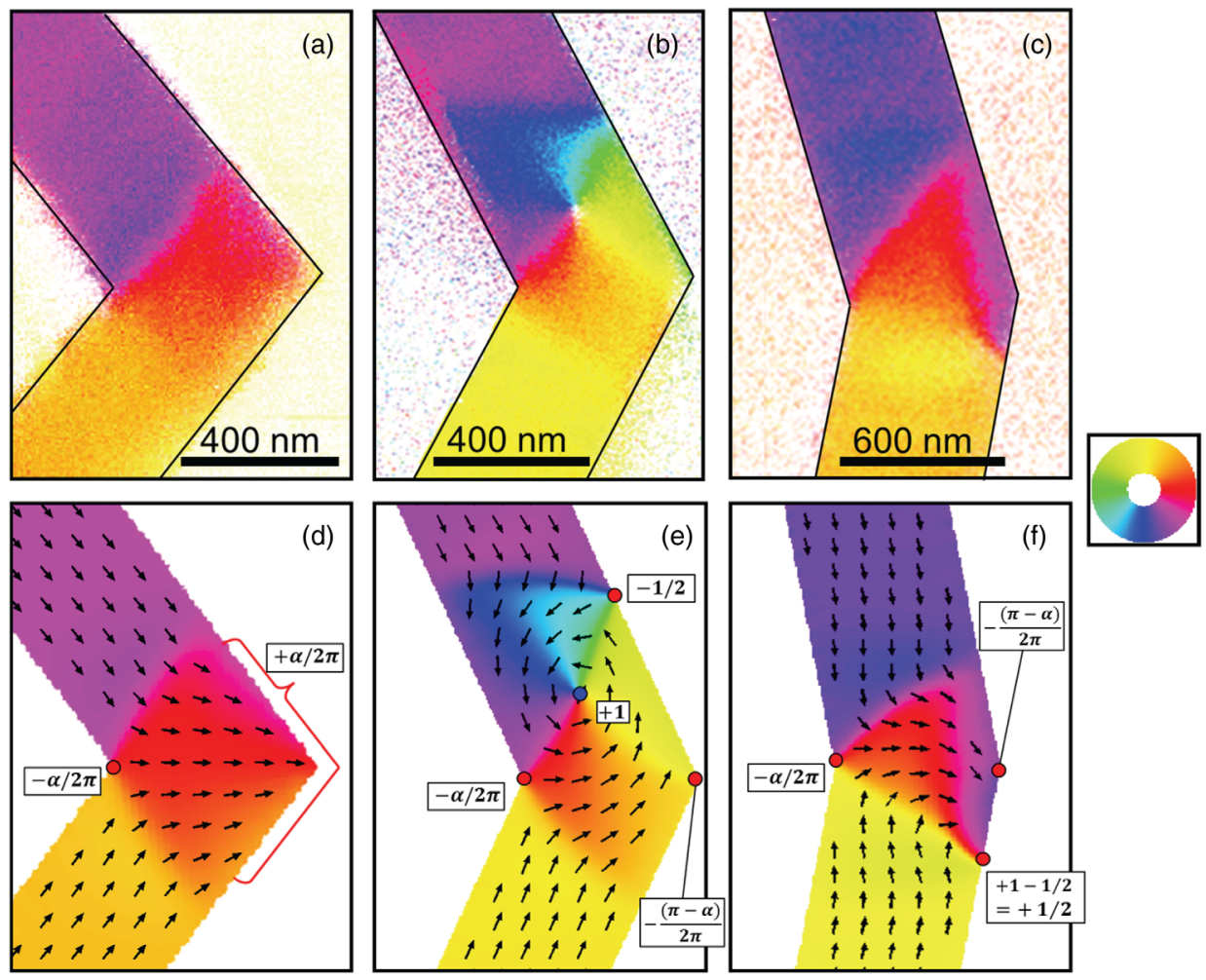

FIG. 4. (a)-(c) SEMPA images and (d)-(f) micromagnetic simulations of the three predominant wall types found for $100 \mathrm{~nm} \leq w \leq 400 \mathrm{~nm}$, $10 \mathrm{~nm} \leq t \leq 30 \mathrm{~nm}$, and $20^{\circ} \leq \alpha \leq$ $160^{\circ}$. The topological points are labeled by red circles (edge defects) and blue circles (volume defects), and the associated topological charge is given next to the circles. (a),(d) Transverse wall in a nanowire with $\alpha=100^{\circ}$, $w=400 \mathrm{~nm}, t=20 \mathrm{~nm}$; (b),(e) vortex wall in a nanowire with $\alpha=120^{\circ}$, $w=400 \mathrm{~nm}, t=20 \mathrm{~nm}$; and (c),(f) asymmetric transverse wall in a nanowire with $\alpha=150^{\circ}, w=600 \mathrm{~nm}$, $t=10 \mathrm{~nm}$. The orientation of magnetization is color coded according to the color wheel. 
[18]. The complex microstructure of the walls is highly reproducible in the experiments, which proves the stability of these structures. A theoretical description of these structures is based on topology [18,55-57], which reduces the micromagnetic structures to their essential features. Based on the topological features, an analytical model is established and discussed in Sec. IV D.

In a straight wire, the transverse wall consists of $a-1 / 2$ edge defect where two Néel walls start with an angle of $90^{\circ}$. These walls enclose a triangular-shaped domain whose magnetization is oriented perpendicular to the magnetization in the wire arms. The opposite $+1 / 2$ defect is widened up to a length of approximately $2 w$. In the magnetostatic limit, the total charge of the transverse wall of $2 M_{S} t w$ is located at the outer edge [18].

In the case of bent wires, the energy of the transverse wall is reduced when the negative edge defect is located at the inner kink, mainly due to the reduction of stray-field energy as the angle between the magnetization and wire edge is reduced. The bend reduces the Néel wall angle from $90^{\circ}$ to $90^{\circ}-\alpha / 2$ and the total angle of magnetization rotation from $180^{\circ}$ to $180^{\circ}-\alpha$. In topological terms, the absolute value of the topological charge, or winding number [57], of both edge defects is reduced compared to the straight wire geometry.

The details of Fig. 4(a), however, reveal that the magnetostatic limit provides a rough picture of the actual distribution of magnetization only. Except at the wire tip, the magnetization in the vicinity of the wire edges is aligned parallel to the edges, which is caused by the high demagnetization field there $[17,58]$. In the micromagnetic simulation, these details are well reproduced [Fig. 4(d)], which indicates that the parameters describe the ferromagnetic material very well. The main features of the fine structure of the transverse wall are retained down to small bending angles (see Fig. 8 below).

Within the topological approach, the vortex wall [Fig. 4(b)] is the state with the second-lowest number of topological defects (in straight wires, two $-1 / 2$ edge defects and one +1 defect, i.e., the vortex core). The magnetic structure of the vortex wall is described in detail for a bent wire elsewhere [25]. Using the same argument as above, it is straightforward to understand that in the case of a V-shaped wire the bend also acts as an attractive potential for the vortex wall when one of the two negative edge defects is located at the inner kink [25]. An important detail of the vortex wall, which has not been investigated yet, is the exact position of the vortex core. For symmetry reasons, the vortex core is located at the center line in a straight wire. For bent wires, however, we observe a slight shift of the core towards the outer edge. The rotation of magnetization matches the shape of the outer edge near the kink. Vice versa, near the inner kink the vortex structure requires more space to adapt to the shape of the edge. The latter reasons cause a core displacement towards the outer edge. For a thickness of $20 \mathrm{~nm}$ and a bending angle of $150^{\circ}$, the average position of the core is moved out of the center line $(0.5 \times w)$ and located at $(0.40 \pm 0.03) \times w$, which is in good agreement with the result from simulations, i.e., $(0.46 \times w)$ [see Figs. 4(b) and 4(e)]. Additionally, the simulations give an approximately linear dependence of the displacement of the core from the center line according to $\{0.35+0.0125[(\alpha-$ $\left.\left.\left.60^{\circ}\right) / 10^{\circ}\right]\right\} w$ in the range $60^{\circ} \leq \alpha \leq 180^{\circ}(t=20 \mathrm{~nm})$.

In contrast to the straight wire, an additional Néel-like wall appears in the bent wire that connects the tip and the vortex core (see Fig. 10 below). Hence, an additional edge defect exists at the tip with the topological charge of $-\left(180^{\circ}-\alpha\right) / 360^{\circ}$ [see Figs. 4(e) and 4(f)]. This feature is important in the estimation of the energy difference between the vortex and transverse wall given in Sec. IV D.

For straight wires, a second position of the +1 defect close to the edge is possible [18]. Beyond the magnetostatic limit, this gives the asymmetric transverse wall which was introduced as the third stable domain-wall type in straight wires based on OOMMF simulations $[17,19]$. The authors predict the asymmetric transverse wall as a metastable state in a very narrow region in the phase diagram of straight wires where the vortex and transverse wall are nearly equal in energy. In our investigation, we find the asymmetric transverse wall in bent nanowires also [Fig. 4(c)]. Although the magnetic fine structure in Fig. 4(c) does not reveal any +1 defect, the overall structure of the wall in the bent nanowire is very similar to that of a vortex wall. The center of the wall is shifted from the axis of symmetry towards a wire arm, so that two mirror-symmetric configurations are possible for a head-to-head domain configuration [19], i.e., the asymmetric transverse wall with an upward or downward inclination of the magnetization at the outer edge. The close connection of the asymmetric transverse wall and vortex wall means that the scheme of the vortex wall formation should also work for the asymmetric transverse wall [25]. This means that the exact orientation of the seeding field determines the position of the center and sense of rotation. In contrast to the vortex wall, the nucleation of the vortex core is suppressed in the case of the asymmetric transverse wall, as the expense of exchange energy for such a small vortex is much too high, which has been postulated from theoretical considerations [18]. Hence, the magnetization configuration at the outer edge is similar to that of $a(+1-1 / 2=)+1 / 2$ pointlike edge defect.

For all wall types, the overall matching of the measured fine structure and the results of the OOMMF simulations is very good. The matching is somewhat less pronounced in the case of the transverse wall, which suggests that the latter configuration is more sensitive to structural defects at the edges generated during FIB preparation $[59,60]$.

For wires of width $w \geq 600 \mathrm{~nm}$, borderline structures occur that are similar to the charged wall found in thin films (see Fig. 5). The structure in Fig. 5(a) reminds one of an alternating chain of vortex $(+1$ defect) and antivortex $(-1$ defect) structures, which avoids excessively long charged $180^{\circ}$ walls. In thin films, this pattern is known 


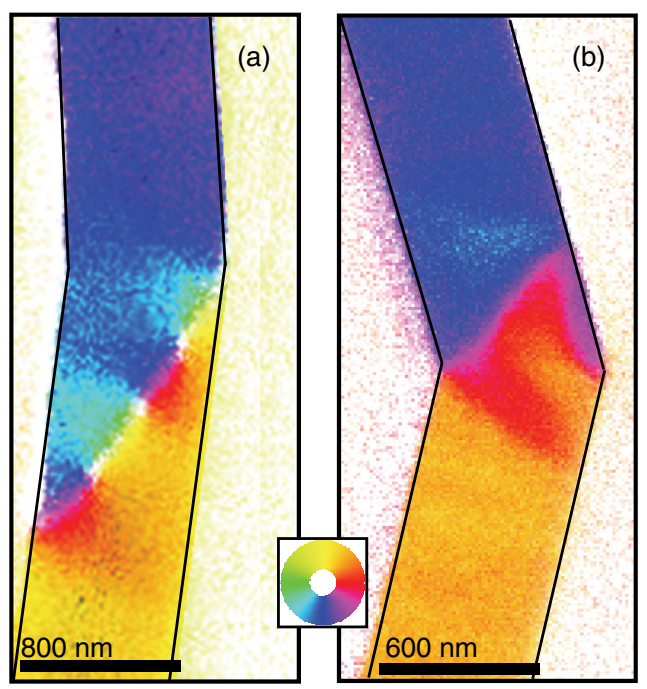

FIG. 5. SEMPA images of domain walls observed in nanowires with widths of $w \geq 600 \mathrm{~nm}$. In this transition region to film (a) cross-tie-like domain walls and (b) zigzaglike domain walls are found, referred to hereinafter as filmlike domain walls. The orientation of magnetization is color coded according to the color wheel.

as a cross-tie wall $[61,62]$. The cross-tie-like structure is observed only in 30-nm-thick wires. In Fig. 5(b), the transition region has a zigzag shape. Similar wall patterns are known to appear in thin films between head-to-head(tail-to-tail-) oriented domains. The zigzag shape of the wall reduces the charge density with respect to the straight wall [63]. As the negative (positive) edge defect is located at the inner (outer) kink of the wire, the structure is very similar to the fine structure of a transverse wall.

\section{B. Impact of wire width and thickness on domain-wall type $\left(\alpha=150^{\circ}\right)$}

In the following, the impact of wire width and thickness on the preponderant type of domain wall is discussed while the bending angle is held constant $\left(\alpha=150^{\circ}\right)$. Figure 6 displays SEMPA images of the magnetic structure for various widths and thicknesses. In thick and wide wires mainly vortex walls are observed, while in thin and narrow wires transverse walls dominate. This is in agreement with the results for straight wires $[7,16]$. In 30-nm-thick wires $\left(\alpha=150^{\circ}\right)$ exclusively vortex walls are found [see Fig. 6(c)] even for a wire width of $w=100 \mathrm{~nm}$, while for $t=20 \mathrm{~nm}$ the transition from vortex to transverse walls is observed below $w=200 \mathrm{~nm}$ [Fig. 6(b)]. In wires with $t=10 \mathrm{~nm}$, vortex walls do not appear at all, while the transverse wall transforms to an asymmetric transverse wall for widths above $600 \mathrm{~nm}$ [Fig. 6(a)]. In the thickness range above $10 \mathrm{~nm}$, wall structures like in thin films are frequently observed in the broad wires (widths $w \geq 600 \mathrm{~nm}$; see Fig. 5).

The thickness- and width-dependent transition between vortex and transverse walls in bent nanowires is simulated with OOMMF $\left(\alpha=150^{\circ}\right)$. The points of equal energy are
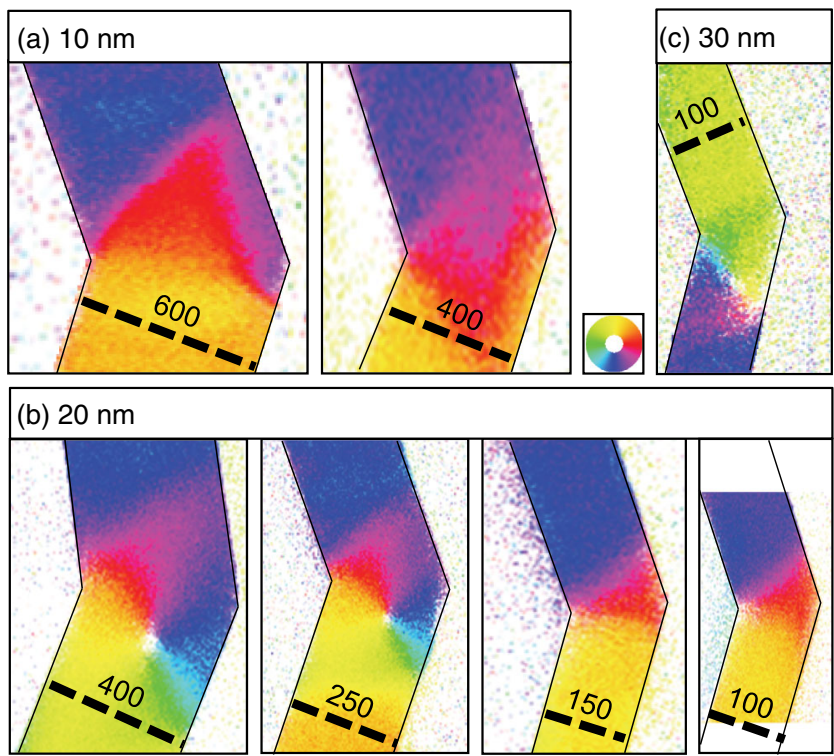

FIG. 6. SEMPA images of domain walls in bent nanowires $\left(\alpha=150^{\circ}\right)$ for different wire widths and thicknesses [(a) 10, (b) 20 , and (c) $30 \mathrm{~nm}$ ]. The wire width is given in the images in nanometers next to the dashed line. The orientation of magnetization is color coded according to the color wheel.

obtained from the simulations and marked by stars in the $t(w)$ diagram (Fig. 7). The line of equal energy satisfies the condition $t(w) \propto 1 / w$ (red line in Fig. 7), as predicted and found for straight wires $[7,16]$. The results of our experimental study are given as symbols: triangles (circles) mark transverse (vortex) walls, while diamonds (squares) represent asymmetric transverse (filmlike) walls, respectively. The general trend obtained from the simulation is in agreement with the experiment. It turns out that the experiments are best resembled if the line of equal energy is shifted towards thicker and wider wires (see the dashed line). This is due to the fact that the transverse wall represents a local minimum in the energy landscape which can be populated, although the vortex wall is still the global energy minimum for the head-to-head arrangement $[7,20]$. Another reason for the shift is connected with deviations from ideal wire edges assumed in the simulation. Because of the fabrication process, inclined edges are produced, and, hence, the strayfield energy of the transverse wall is considerably reduced compared to the case of perfect edges $[59,60]$.

The micromagnetic calculations reveal that the asymmetric transverse wall does not represent the global energy minimum for nanowires in the range of the phase diagram with boundaries: $10 \mathrm{~nm} \leq t \leq 30 \mathrm{~nm}$ and $150 \mathrm{~nm} \leq w \leq$ $400 \mathrm{~nm}$ (for $\alpha=150^{\circ}$ ). Close to the phase boundary between the transverse and vortex wall, the energy of the asymmetric transverse wall is about $2 \%$ higher than the energy of the transverse wall. Small deviations from the ideal wire geometry can influence the relaxation process and stabilize the asymmetric wall. In the straight wire, an area of global minimum close to the phase boundary is identified 


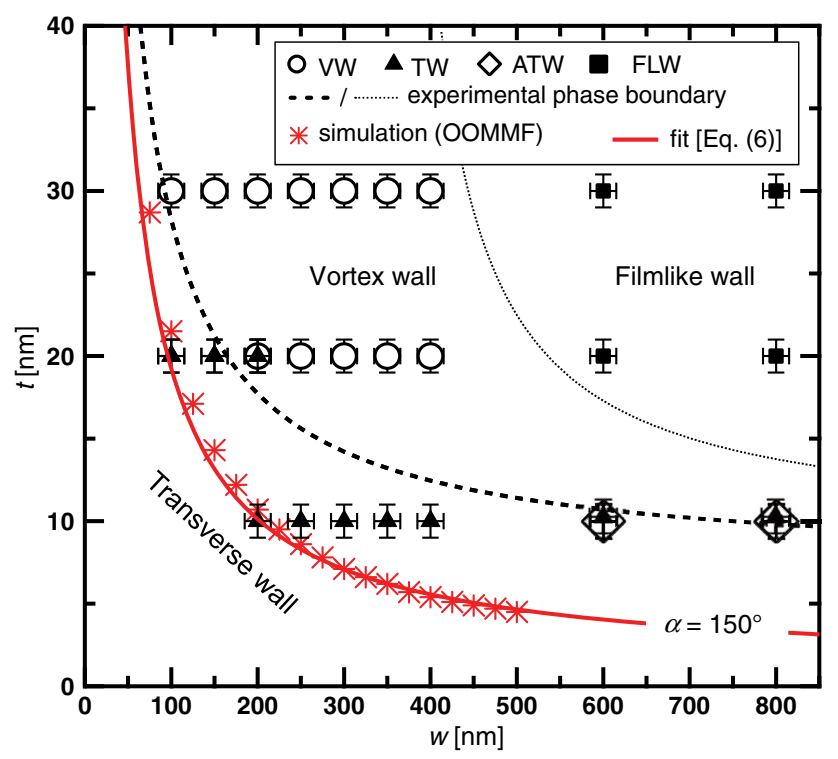

FIG. 7. Phase diagram displaying the wall structure as a function of the thickness and wire width. The bending angle is fixed to $\alpha=150^{\circ}$. The calculated points of equal energy of the transverse and vortex wall (red stars) are shown together with the experimental results, whereby triangles represent transverse walls, circles vortex walls, diamonds asymmetric transverse walls, and squares filmlike walls (see Fig. 6). The fit of the numerical data (red line) is based on Eq. (6) $[t(w) \propto 1 / w]$ and represents the line of equal energy. To guide the eye, we plot similar lines $[t(w) \propto 1 / w]$ for the experimentally found transition between the transverse and vortex wall, and vortex and filmlike wall, respectively.

by numerical methods [17]. In the experiment, the asymmetric wall is infrequently found in the vicinity of the phase boundary for $t>10 \mathrm{~nm}$. In the thinnest wires $(t=10 \mathrm{~nm})$, the asymmetric transverse wall appears to be preponderant for $w \geq 600 \mathrm{~nm}$. This finding indicates that the nucleation of a vortex core is unlikely in such thin wires.

\section{Impact of bending angle and thickness on the type of domain wall $(w=400 \mathrm{~nm})$}

In the former section, the bending angle is kept constant. We now focus on the dependence of the domain-wall type on the thickness and bending angle while the wire width is kept constant. The latter set of parameters will give a second sectional plane through the 3D phase diagram (perpendicular to the one in the last paragraph). The SEMPA micrographs in Fig. 8 show the magnetic microstructure found in wires of constant width $(400 \mathrm{~nm})$ and various bending angles and thicknesses. The general finding is that for obtuse (acute) angles vortex (transverse) walls are found, respectively. Second, the range of angles and widths, in which the transverse wall is dominant, increases with decreasing thickness. In 10-nm-thick wires, no vortex walls are observed at any angle, while a change from the symmetric to asymmetric transverse wall is found in the range of $\alpha=150^{\circ}$ to $160^{\circ}$ [Fig. 8(a)]. In 30-nm-thick wires, vortex walls are observed for bending angles above $\alpha=90^{\circ}$, and below $80^{\circ}$ the transverse wall is dominant [Fig. 8(c)]. In the range of $\alpha=80^{\circ}-90^{\circ}$, the asymmetric transverse wall is predominant. In 20 -nm-thick wires, the transition range is slightly shifted to larger bending angles of $\alpha=110^{\circ}-120^{\circ}$ [see Figs. 8(b) and 9(b)], where all three kinds of wall are found [see the histogram in Fig. 9(a)].

The experimental results are summarized in Fig. 9(b). The line of equal energy between the vortex and transverse wall calculated with OOMMF is indicated by stars. To guide the eye, we plot a dashed line [based on Eq. (6) given below] that indicates the line of equal energy of the

\section{(a) $10 \mathrm{~nm}$}

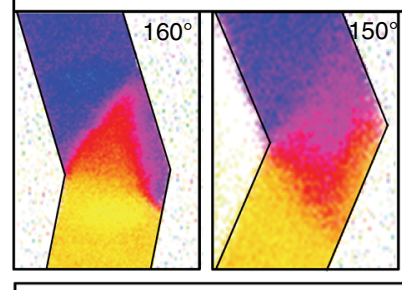

(b) $20 \mathrm{~nm}$

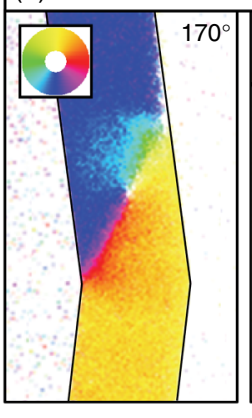

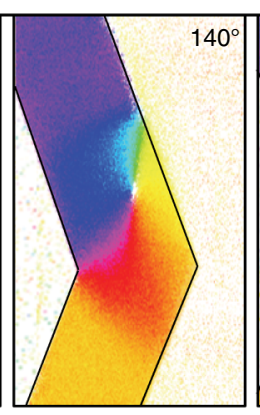

(c) $30 \mathrm{~nm}$
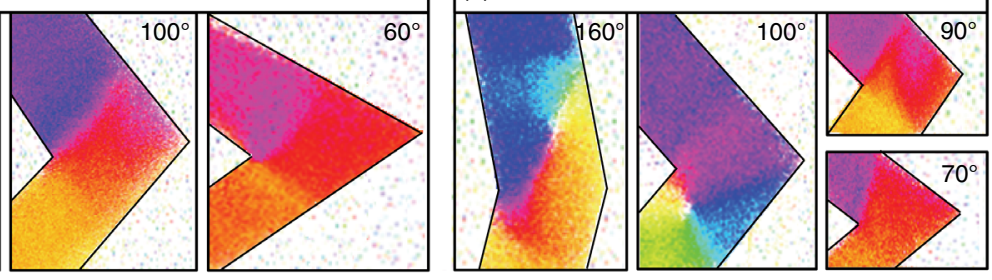

I.

,

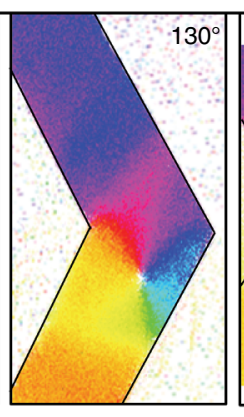

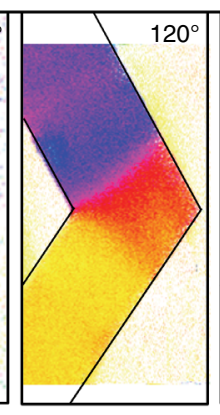
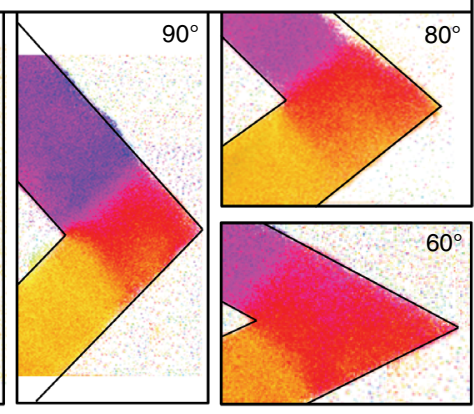

FIG. 8. SEMPA images of domain walls in bent nanowires $(w=400 \mathrm{~nm})$ for different bending angles $\alpha$ and thicknesses [(a) 10, (b) 20, and (c) $30 \mathrm{~nm}$ ]. The bending angles are indicated in the top right corner of the micrographs. The orientation of magnetization is color coded according to the color wheel. The different positions of the vortex core are due to a change of the seeding field orientation. 

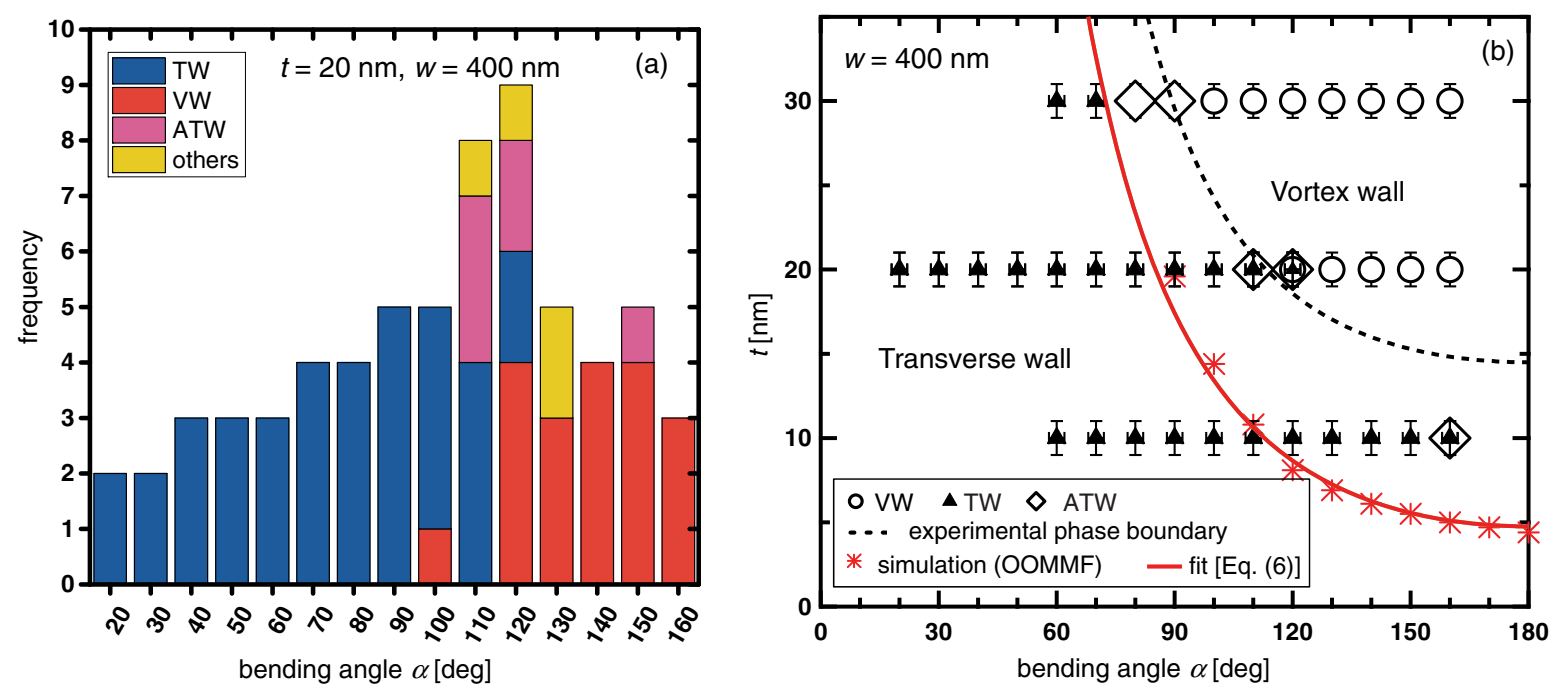

FIG. 9. (a) Histogram of observed wall types as a function of the bending angle for wires with $t=20 \mathrm{~nm}$ and $w=400 \mathrm{~nm}$. "Others" contain zigzag, cross-tie, and not assignable wall types. (b) Phase diagram displaying the domain-wall type as a function of the thickness and bending angle for nanowires with a wire width of $w=400 \mathrm{~nm}$. The experimental results are indicated by triangles for transverse, circles for vortex, and diamonds for asymmetric transverse walls. The simulated points of equal energy of the transverse and vortex wall (red stars) are shown. The red line represents the line of equal energy, which is a fit to the numerical data based on the phenomenological model [Eq. (6)]. The dashed line [also based on Eq. (6)] is a guide to the eye indicating the experimentally found transition between vortex and transverse walls.

experimental results. Again, the simulation and experiment show the same trend. The line of equal energy separates a region of wires with acute angles and transverse walls from a region of wires with obtuse angles and vortex walls. The calculated phase boundary is slightly shifted towards smaller angles. This can be explained following the same arguments as in the previous section. Again, the asymmetric transverse wall is primarily found around the phase boundary but does not represent the global minimum according to the numeric calculations.

The 2D phase diagrams presented in Secs. IV B and IV C can be combined to create a three-dimensional phase diagram. In the 3D diagram with the parameters wire width, thickness, and bending angle, the points of equal energy between the vortex and transverse domain wall build a plane of equal energy (see Fig. 13 below). In the following section, a phenomenological model is derived that describes the plane of equal energy as a function of the geometric parameters.

\section{Phenomenological description of the plane of equal energy between transverse and vortex wall}

The starting point for the development of a phenomenological model is the study for straight wires by McMichael and Donahue [16]. In the following, the focus is put on energy differences between the transverse and vortex wall. Considering energy differences means that the energy of the Neél walls which start at the inner kink can be neglected, as they appear and are comparable in both microstructures (for a consideration of the topological +1 defect of the vortex structure, see below). Hence, the energy contribution of the transverse wall that matters for the comparison of energies is the stray-field energy. In order to illustrate the validity of the approximation, $(\nabla \times \mathbf{M})_{z}$ is shown in Fig. 10 for straight and bent wire geometry.

Besides the vortex core, the microstructure of the vortex wall has additional Néel-like walls that have to be considered in the comparison of energies. One wall is located in the wire arm [see the wall located at the bottom in Fig. 10(e)]. Note that for arbitrary bending angles this wall remains the same due to topological reasons. More explicitly, the wall always has to be a $90^{\circ}$ Néel wall at the wire edge where the wall starts as the topological charge of the edge defect is $-1 / 2$. The wall appears also in a straight wire [Fig. 10(b)] but is not considered in the model of Ref. [16]. In case the wire is bent, a further Néel-like wall forms near the outer kink [see the black dashed line in Fig. 10(e)].

Following the above considerations, the additional energy of the vortex wall compared to the transverse wall comes from the vortex core, a $90^{\circ}$ Néel wall, and a Néel-like wall in the vicinity of the tip. While the former Néel wall does not depend on wire bending, the latter does. The rotation of magnetization in the vicinity of the tip is $180^{\circ}-\alpha$ [see Fig. 4(e)]. Hence, the energy of the wall at the tip $E_{\text {Tip Néel wall }}$ can be approximated for a wall of volume $t \times a \times b$ as

$$
\begin{aligned}
E_{\text {Tip Néel wall }}(t, w, \alpha) & =C_{1} t w / \sin (\alpha / 2) \int_{0}^{b}\left(\frac{d \phi}{d x}\right)^{2} d x \\
& =C_{1} t w / \sin (\alpha / 2)\left(180^{\circ}-\alpha\right)^{2}
\end{aligned}
$$



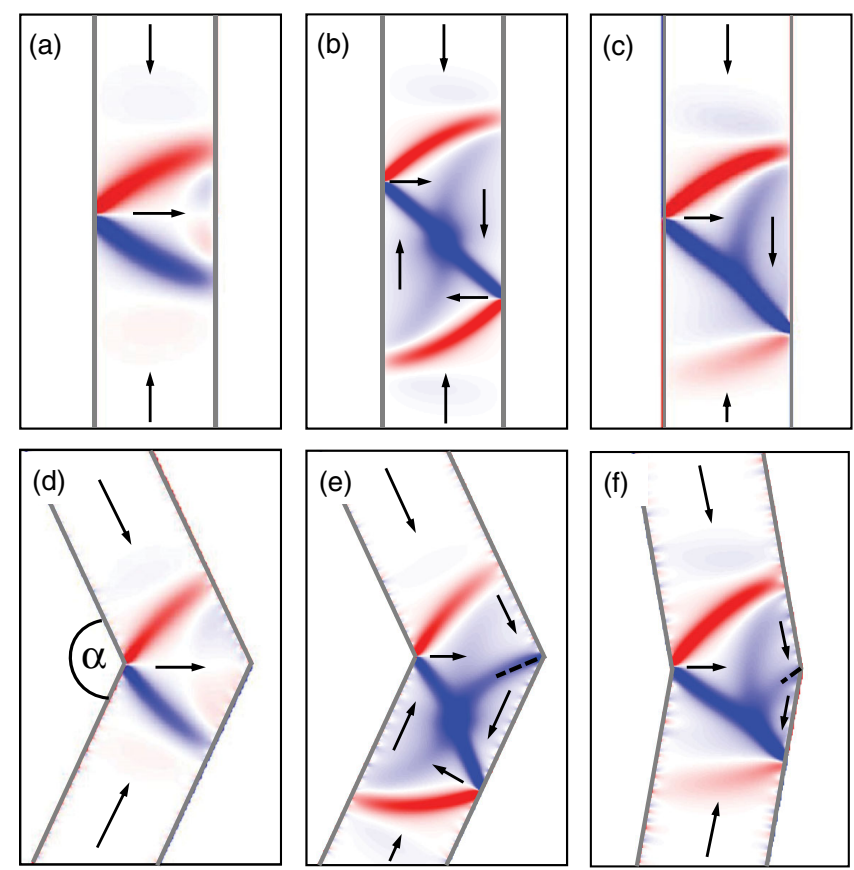

FIG. 10. Micromagnetic calculation of the curl of the magnetization $(\nabla \times \mathbf{M})_{z}$ for different wall types in straight (a)-(c) and bent (d)-(f) nanowires. (a),(d) Transverse wall; (b),(e) vortex wall; (c),(f) asymmetric transverse wall. The arrows indicate the magnetization orientation, while the dashed lines highlight the additional Néel wall in the bent wires for the vortex and asymmetric transverse walls.

with $C_{1}$ being a factor of proportionality standing for the energy per wall area and $\operatorname{rad}^{2} . \phi$ stands for the orientation of magnetization within the wall with width $b$. The wall length $a$ is assumed to be proportional to the cross section of the wire at the bisection, i.e., $w / \sin (\alpha / 2)$ [see the dashed line in Fig. 10(e)]. Further simplifications are (a) the wall width $b$ is independent on the parameters of the wire and (b) $d \phi / d x=\left(180^{\circ}-\alpha\right) / b$ is uniform in the wall [63]. It is worth mentioning that the additional Néel wall is also present in the case of the asymmetric transverse wall in a bent wire [compare Figs. 10(e) and 10(f)], which again reveals its close relationship to the vortex wall.

The domain-wall energy of the $90^{\circ}$ Néel wall associated with the $-1 / 2$ edge defect located in the wire arm [see Fig. 4(e)] is given by

$$
E_{90^{\circ} \text { Néel wall }}(t, w, \alpha)=C_{2} t w,
$$

where $C_{2}$ is a further constant associated with the energy per wall area of a $90^{\circ}$ Néel wall. Following the description given by McMichael and Donahue, the energy of the vortex core can be approximated as

$$
E_{\text {core }}(t, w, \alpha)=2 \pi t A \ln (\pi) \approx 7.2 A t,
$$

where $A$ is the exchange stiffness [16]. The vortex core is located in one of the wire arms, and the surrounding of the core retains the same microstructure as in a straight wire, so that it is reasonable to assume that $E_{\text {core }}$ is not significantly influenced by the bend.

Finally, an estimation of the stray-field energy difference connected with surface charges that is associated with the triangular-shaped domain in a transverse wall has to be worked out. For a straight wire, the stray-field energy is given as $E_{\mathrm{SF}} \approx 1 / 8 \mu_{0} M_{s}{ }^{2} t^{2} w$ [16]. Comparing the transverse wall in the straight wire [Fig. 10(a)] with the one in the bent wire [Fig. 10(d)], it can be seen that the fine structure has not significantly changed, while the magnetization within the triangular-shaped domain $\mathbf{M}_{\mathrm{TW}}$ exhibits an angle of $\alpha / 2$ to the magnetization in the adjacent wire sections. The change of stray-field energy with the bending angle comes from the reduction of the pole strength $\sigma$ due to the tilt of the magnetization $\mathbf{M}_{\mathrm{TW}}$ with respect to the normal of the wire edge $\sigma=\mathbf{M}_{\mathrm{TW}} \cdot \mathbf{n}=M_{S} \sin (\alpha / 2)$, whereby $\mathbf{n}$ denotes the normal vector on the wire arm. Hence, the demagnetization field is $H_{d}=1 / 2 M_{S} \sin (\alpha / 2)$. When additionally taking into account that the length of the charged surface of the wire depends on the bending angle $[2 w / \sin (\alpha / 2)]$, as a consequence of the conservation of magnetic flux, this finally yields the angle-dependent strayfield energy $E_{\mathrm{SF}}$ of the transverse wall:

$$
E_{\mathrm{SF}}(t, w, \alpha) \approx C_{0} / 8 \mu_{0} M_{S}^{2} \sin (\alpha / 2) t^{2} w .
$$

In the limiting case $\alpha \rightarrow 0$, the stray-field energy of the transverse wall $E_{\mathrm{SF}}$ vanishes. $C_{0}$ is a constant of approximately 1 . Small deviations from $C_{0}=1$ are possible and due to a simplified assumption about the charge distribution at the edges [16].

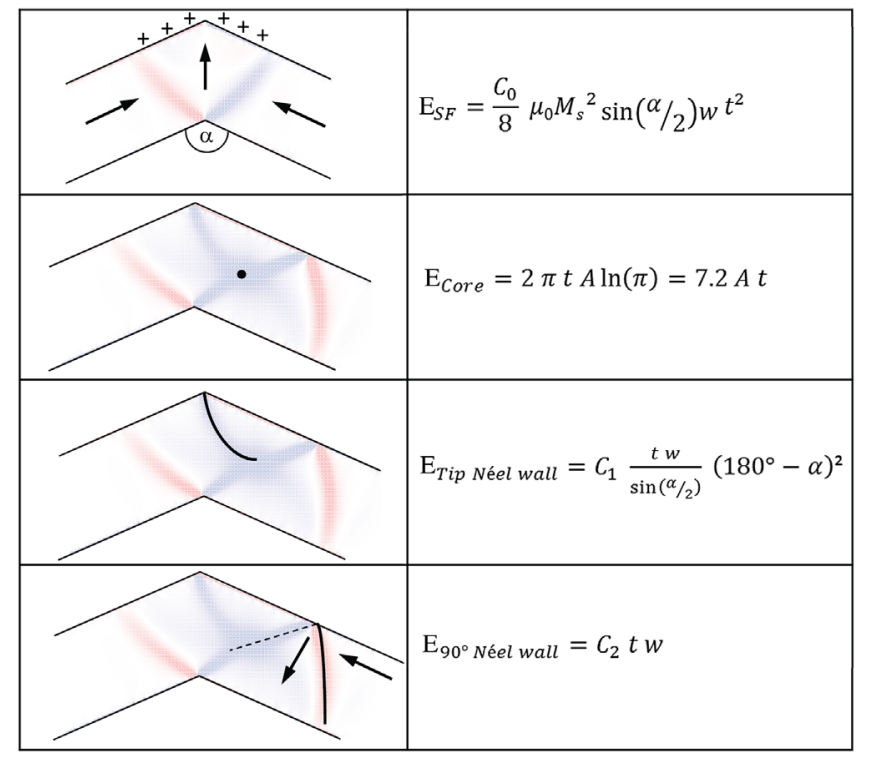

Fig. 11. Sketch and corresponding energy contributions of the important differences between the transverse wall compared to the vortex wall (upper panel) and vice versa (three lower panels). For explanations, see the text. 


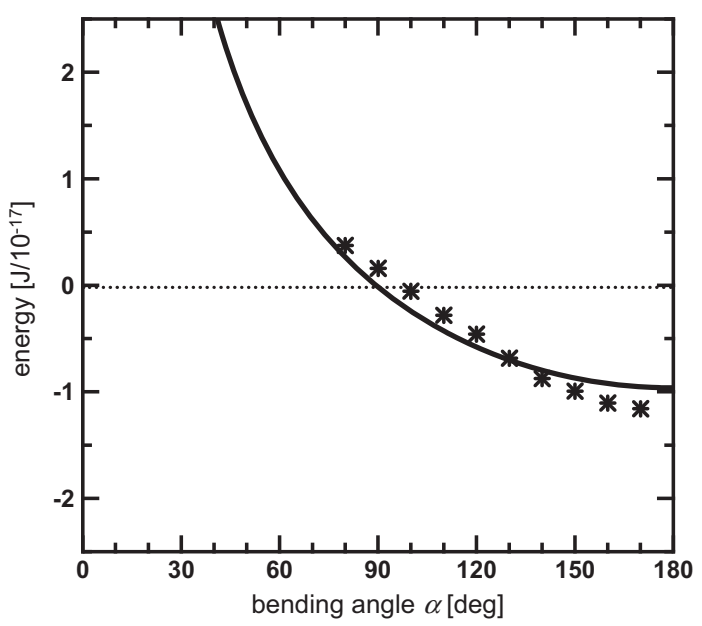

FIG. 12. Numerically calculated (OOMMF) total energy differences between the transverse wall and vortex wall in dependence of the bending angle for a nanowire of width $w=$ $250 \mathrm{~nm}$ and thickness $t=20 \mathrm{~nm}$. The solid line represents the plot utilizing Eq. (5) and the obtained fit parameters.

The four energy terms that play a role in the energy difference between the vortex and transverse wall are summarized and sketched in Fig. 11. The functional relation for the plane of equal energy between the vortex and transverse wall follows from $\Delta E_{\text {diff }}=0$, where $\Delta E_{\text {diff }}=E_{\mathrm{VW}}-E_{\mathrm{TW}}$ is given by [using Eqs. (1)-(4)]

$$
\begin{aligned}
\Delta E_{\text {diff }}= & 7.2 A \mathrm{t}+C_{1} t w / \sin (\alpha / 2)\left(180^{\circ}-\alpha\right)^{2}+C_{2} t w \\
& -C_{0} / 8 \mu_{0} M_{S}^{2} \sin (\alpha / 2) t^{2} w .
\end{aligned}
$$

In order to check the validity of the model, it is used to describe the plane of equal energy $\Delta E_{\text {diff }}(t, w, \alpha)=0$ that is numerically calculated (OOMMF) utilizing the material constants of $\mathrm{Co}_{39} \mathrm{Fe}_{54} \mathrm{Si}_{7}\left(\mu_{0} M_{S}=1.8 \mathrm{~T}, A=35 \mathrm{pJ} / \mathrm{m}\right)$.

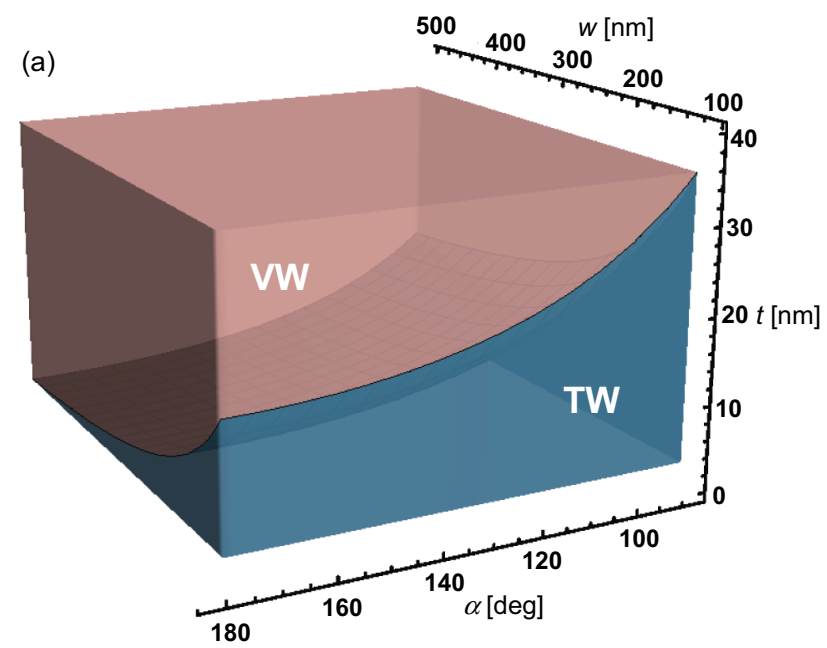

For that purpose, the lines of equal energy in the $t(w)$ diagram (Fig. 7, constant bending angle $\alpha=150^{\circ}$ ) and the $t(\alpha)$ diagram (Fig. 9, $w=400 \mathrm{~nm}$ ) are simultaneously fitted to

$$
\begin{aligned}
& \left.t(w, \alpha)\right|_{\Delta \mathrm{E}_{\mathrm{diff}}(t, w, \alpha)=0} \\
& =\left\{7.2 A+C_{1} w / \sin (\alpha / 2)(180-\alpha)^{2}\right. \\
& \left.+C_{2} w\right\} /\left[C_{0} / 8 \mu_{0} M_{S}^{2} \sin (\alpha / 2) w\right] .
\end{aligned}
$$

As a result, a good accordance of the fit (red lines) and data points is obtained. Hence, the proposed model seems to cover the relevant differences in energy between the vortex and transverse wall as a function of the bending angle and width. The fit parameters are $C_{0}=(0.8 \pm 0.1), C_{1}=$ $(3.1 \pm 0.3) \times 10^{-4} \mathrm{~J} /\left(\mathrm{m}^{2} \mathrm{rad}^{2}\right)$, and $C_{2}=(5.0 \pm 1.0) \times$ $10^{-5} \mathrm{~J} / \mathrm{m}^{2} . C_{0}$ is close to one, confirming the validity of the used ansatz for the stray-field energy connected with the surface charges of the transverse wall. For $\alpha \rightarrow 0^{\circ}$, the expression $C_{1}\left(180^{\circ}-\alpha\right)^{2}$ gives a value of $3.1 \times 10^{-3} \mathrm{~J} / \mathrm{m}^{2}$, which should correspond to the energy of a $180^{\circ}$ Néel wall, i.e., $\gamma_{180^{\circ}}=4 \sqrt{\left[\left(A \mu_{0} M_{S}^{2}\right) / 2\right]}=26.8 \times 10^{-3} \mathrm{~J} / \mathrm{m}^{2}$ [63]. The lower value of the fit can be explained by the fact that the wall angle gradually decreases from the edge towards the vortex core. The same argument explains the value of $C_{2}$ that is much smaller than the energy of a $90^{\circ}$ Néel wall given by $\gamma_{90^{\circ}}=0.25 \cdot \gamma_{180^{\circ}}=6.7 \times 10^{-3} \mathrm{~J} / \mathrm{m}^{2}[63]$.

In order to check whether the model is capable of describing the energy difference between the transverse and vortex wall far from equilibrium, the numerically calculated curve $\Delta E_{\text {diff }}(\alpha)$ is compared to the corresponding model curve using the fit parameters from above for lateral dimensions of $w=250 \mathrm{~nm}$ and $t=20 \mathrm{~nm}$ (without any adjustable parameters; see Fig. 12). Obviously, all features of the numerically calculated curve are well reproduced by the model.

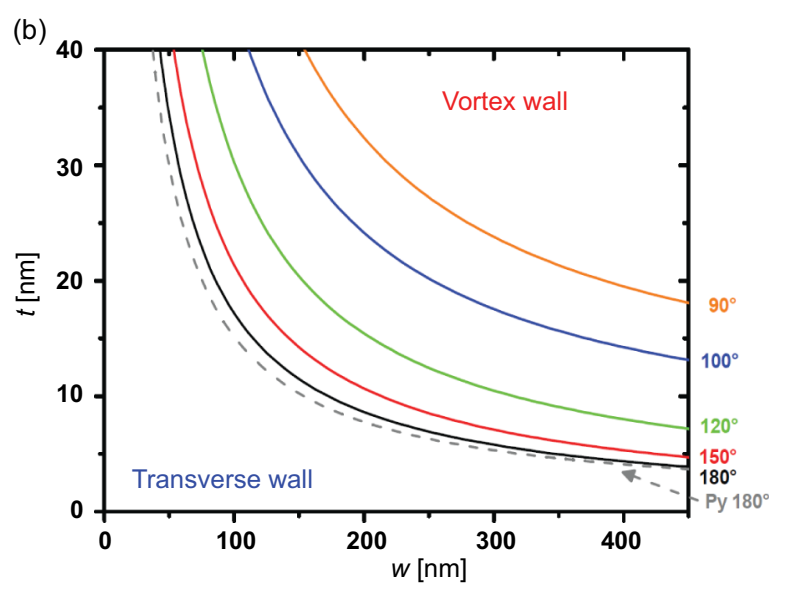

FIG.13. Phase diagrams. (a) Phase diagram displaying the global energy minimum of wall type as a function of the geometric parameters $t, w$, and $\alpha$. The diagram is created utilizing Eq. (6) and the obtained fit parameters (see the text). (b) For selected angles $\alpha$, the line of equal energy is shown in the $t(w)$ diagram. For the sake of comparison with calculations for permalloy, the results for the straight permalloy wire taken from Ref. [12] are added. 
Finally, the plane of equal energy in a three-parameter plot $(t, w, \alpha)$ is shown in Fig. 13(a), utilizing Eq. (6) and the fit parameters. For a given bending angle, the energetic degeneracy of the vortex and transverse wall is found for the values $w t \approx$ constant [Fig. 13(b)]. The latter is also found for straight wires. For comparison, the result for the straight permalloy wire taken from Ref. [17] is shown. Obviously, the results for permalloy and $\mathrm{Co}_{39} \mathrm{Fe}_{54} \mathrm{Si}_{7}$ agree quite well, which confirms our assertion that the results of the present paper can be applied straightforwardly to permalloy.

\section{CONCLUSION}

The impact of geometrical parameters on the magnetic fine structure of symmetric transverse, asymmetric transverse, and vortex walls which are pinned at the kink of softmagnetic V-shaped nanowires is imaged and discussed. The SEMPA measurements reveal details of the microstructure that are beyond the simple domain theoretical limit. For the vortex wall, an additional Néel wall is found that connects the vortex core and tip of the wire. It is further demonstrated that the vortex core is moved outward from the center line of the wire. The asymmetric transverse wall is observed in bent nanowires, and a tight micromagnetic connection to the vortex wall is proven. The preponderant type of domain wall as a function of the geometrical parameters reveals that, besides the thickness and width, the bending angle is an important parameter to tune the remanent wall type on purpose. In the case of wide and thick wires with obtuse angles the vortex wall is the global minimum, whereas in the case of narrow and thin wires with acute angles the transverse wall is favored. Based on the dominant features of the vortex and transverse wall, a phenomenological model is developed that describes the plane of equal energy between both types of walls as a function of geometric parameters. The modeling reproduces nicely the features that are found in the experiment and micromagnetic simulation. As the magnetostatic exchange lengths of $\mathrm{Co}_{39} \mathrm{Fe}_{54} \mathrm{Si}_{7}$ and permalloy are similar, the results of the present investigation can be applied for permalloy wires to a good approximation.

\section{ACKNOWLEDGMENTS}

We gratefully acknowledge financial support by DFG via SFB 668. We thank J. Topp for performing the FMR measurements.

[1] J.-P. Tetienne, T. Hingant, J.-V. Kim, L. Herrera Diez, J.-P. Adam, K. Garcia, J.-F. Roch, S. Rohart, A. Thiaville, D. Ravelosona, and V. Jacques, Nanoscale imaging and control of domain-wall hopping with a nitrogen-vacancy center microscope, Science 344, 1366 (2014).
[2] A. Bisig, M.-A. Mawass, M. Stärk, C. Moutafis, J. Rhensius, J. Heidler, S. Gliga, M. Weigand, T. Tyliszczak, B. Van Waeyenberge, H. Stoll, G. Schütz, and M. Kläui, Dynamic domain wall chirality rectification by rotating magnetic fields, Appl. Phys. Lett. 106, 122401 (2015).

[3] P. S. Keatley, W. Yu, L. O’Brien, D. E. Read, R. P. Cowburn, and R. J. Hicken, Time-resolved Kerr microscopy of coupled transverse domain walls in a pair of curved nanowires, J. Appl. Phys. 115, 17D507 (2014).

[4] J. Leliaert, B. Van de Wiele, A. Vansteenkiste, L. Laurson, G. Durin, L. Dupré, and B. Van Waeyenberge, Influence of material defects on current-driven vortex domain wall mobility, Phys. Rev. B 89, 064419 (2014).

[5] V. Estévez and L. Laurson, Magnetic domain-wall dynamics in wide permalloy strips, Phys. Rev. B 93, 064403 (2016).

[6] R. Allenspach and P.-O. Jubert, Magnetic domain walls in nanowires, MRS Bull. 31, 395 (2006).

[7] M. Kläui, Head-to-head domain walls in magnetic nanostructures, J. Phys. Condens. Matter 20, 313001 (2008).

[8] V. Estévez and L. Laurson, Head-to-head domain wall structures in wide permalloy strips, Phys. Rev. B 91, 054407 (2015).

[9] L. Döring, C. Hengst, F. Otto, and R. Schäfer, Interacting tails of asymmetric domain walls: Theory and experiments, Phys. Rev. B 93, 024414 (2016).

[10] P. J. Metaxas, M. Albert, S. Lequeux, V. Cros, J. Grollier, P. Bortolotti, A. Anane, and H. Fangohr, Resonant translational, breathing, and twisting modes of transverse magnetic domain walls pinned at notches, Phys. Rev. B 93, 054414 (2016).

[11] A. Pivano and V. O. Dolocan, Chaotic dynamics of magnetic domain walls in nanowires, Phys. Rev. B 93, 144410 (2016).

[12] V. Raposo, S. Moretti, M. A. Hernandez, and E. Martinez, Domain wall dynamics along curved strips under current pulses: The influence of Joule heating, Appl. Phys. Lett. 108, 042405 (2016).

[13] S. S. P. Parkin, M. Hayashi, and L. Thomas, Magnetic domain-wall racetrack memory, Science 320, 190 (2008).

[14] M. Diegel, R. Mattheis, and E. Halder, $360^{\circ}$ domain wall investigation for sensor applications, IEEE Trans. Magn. 40, 2655 (2004).

[15] J. Vandermeulen, B. Van de Wiele, L. Dupré, and B. Van Waeyenberge, Logic and memory concepts for allmagnetic computing based on transverse domain walls, J. Phys. D 48, 275003 (2015).

[16] R. McMichael and M. Donahue, Head to head domain wall structures in thin magnetic strips, IEEE Trans. Magn. 33, 4167 (1997).

[17] Y. Nakatani, A. Thiaville, and J. Miltat, Head-to-head domain walls in soft nano-strips: A refined phase diagram, J. Magn. Magn. Mater. 290-291, 750 (2005).

[18] H. Youk, G.-W. Chern, K. Merit, B. Oppenheimer, and O. Tchernyshyov, Composite domain walls in flat nanomagnets: The magnetostatic limit, J. Appl. Phys. 99, 08B101 (2006).

[19] A. Thiaville, Y. Nakatani, F. Piéchon, J. Miltat, and T. Ono, Transient domain wall displacement under spin-polarized current pulses, Eur. Phys. J. B 60, 15 (2007).

[20] M. Kläui, C. A. F. Vaz, J. A. C. Bland, L. J. Heyderman, F. Nolting, A. Pavlovska, E. Bauer, S. Cherifi, S. Heun, 
and A. Locatelli, Head-to-head domain-wall phase diagram in mesoscopic ring magnets, Appl. Phys. Lett. 85, 5637 (2004).

[21] D. Backes, C. Schieback, M. Kläui, F. Junginger, H. Ehrke, P. Nielaba, U. Ruediger, L. J. Heyderman, C. S. Chen, T. Kasama, R. E. Dunin-Borkowski, C. A. F. Vaz, and J. A. C. Bland, Transverse domain walls in nanoconstrictions, Appl. Phys. Lett. 91, 112502 (2007).

[22] C. W. Sandweg, N. Wiese, D. McGrouther, S. J. Hermsdoerfer, H. Schultheiss, B. Leven, S. McVitie, B. Hillebrands, and J.N. Chapman, Direct observation of domain wall structures in curved permalloy wires containing an antinotch, J. Appl. Phys. 103, 093906 (2008).

[23] M. Kläui, H. Ehrke, U. Rüdiger, T. Kasama, R. E. DuninBorkowski, D. Backes, L. J. Heyderman, C. A. F. Vaz, J. A. C. Bland, G. Faini, E. Cambril, and W. Wernsdorfer, Direct observation of domain-wall pinning at nanoscale constrictions, Appl. Phys. Lett. 87, 102509 (2005).

[24] T. Taniyama, I. Nakatani, T. Namikawa, and Y. Yamazaki, Resistivity due to Domain Walls in Co Zigzag Wires, Phys. Rev. Lett. 82, 2780 (1999).

[25] S. Hankemeier, A. Kobs, R. Frömter, and H. P. Oepen, Controlling the properties of vortex domain walls via magnetic seeding fields, Phys. Rev. B 82, 064414 (2010).

[26] P. O. Jubert, M. Kläui, A. Bischof, U. Rüdiger, and R. Allenspach, Velocity of vortex walls moved by current, J. Appl. Phys. 99, 08 G523 (2006).

[27] A. Vanhaverbeke, A. Bischof, and R. Allenspach, Control of Domain Wall Polarity by Current Pulses, Phys. Rev. Lett. 101, 107202 (2008).

[28] L. Heyne, M. Kläui, D. Backes, P. Möhrke, T. a. Moore, J. G. Kimling, O. Boulle, U. Rüdiger, L. J. Heyderman, a. F. Rodriguez, F. Nolting, K. Kirsch, and R. Mattheis, Direct imaging of current-induced domain wall motion in $\mathrm{CoFeB}$ structures, J. Appl. Phys. 103, 07D928 (2008).

[29] M. Kläui, P. O. Jubert, R. Allenspach, A. Bischof, J. A. C. Bland, G. Faini, U. Rüdiger, C. A. F. Vaz, L. Vila, and C. Vouille, Direct Observation of Domain-Wall Configurations Transformed by Spin Currents, Phys. Rev. Lett. 95, 026601 (2005).

[30] M. Hayashi, L. Thomas, Ya. B. Bazaliy, C. Rettner, R. Moriya, X. Jiang, and S. S. P. Parkin, Influence of Current on Field-Driven Domain Wall Motion in Permalloy Nanowires from Time Resolved Measurements of Anisotropic Magnetoresistance, Phys. Rev. Lett. 96, 197207 (2006).

[31] J. L. Tsai, S. F. Lee, Y. D. Yao, C. Yu, and S. H. Liou, Magnetoresistance study in thin zig zag NiFe wires, J. Appl. Phys. 91, 7983 (2002).

[32] M. Tanase, D. M. Silevitch, C. L. Chien, and D. H. Reich, Magnetotransport properties of bent ferromagnetic nanowires, J. Appl. Phys. 93, 7616 (2003).

[33] L. K. Bogartand and D. Atkinson, Magnetotransport properties of bent ferromagnetic nanowires, Appl. Phys. Lett. 94, 042511 (2009).

[34] L. O’Brien, D. Petit, H. T. Zeng, E. R. Lewis, J. Sampaio, A. V. Jausovec, D. E. Read, and R. P. Cowburn, Near-Field Interaction between Domain Walls in Adjacent Permalloy Nanowires, Phys. Rev. Lett. 103, 077206 (2009).

[35] E. S. Wilhelm, D. McGrouther, L. Heyne, A. Bisig, and M. Kläui, Vortex domain wall chirality rectification due to the interaction with end domain spin structures in permalloy nanowires, Appl. Phys. Lett. 95, 252501 (2009).

[36] M. Donolato, F. Lofink, S. Hankemeier, J. M. Porro, H. P. Oepen, and P. Vavassori, Characterization of domain wall-based traps for magnetic beads separation, J. Appl. Phys. 111, 07B336 (2012).

[37] H. P. Oepen and H. Hopster, Magnetic Microscopy of Nanostructures (Springer-Verlag, Berlin, 2005), ISBN 3540401865.

[38] F. Lofink, S. Hankemeier, R. Frömter, J. Kirschner, and H. P. Oepen, Long-time stability of a low-energy electron diffraction spin polarization analyzer for magnetic imaging, Rev. Sci. Instrum. 83, 023708 (2012).

[39] A. Berger and H.P. Oepen, Magnetic domain walls in ultrathin fcc cobalt films, Phys. Rev. B 45, 12596 (1992).

[40] R. Frömter, S. Hankemeier, H. P. Oepen, and J. Kirschner, Optimizing a low-energy electron diffraction spinpolarization analyzer for imaging of magnetic surface structures, Rev. Sci. Instrum. 82, 033704 (2011).

[41] M. Donahue and D. Porter, Interagency Report NISTIR 6376, National Institute of Standards and Technology, 1999.

[42] Preliminary results for $t=10 \mathrm{~nm}$ and $w=100 \mathrm{~nm}$ show transverse walls that do not pin at the bend but at arbitrary positions within the wire. Moreover, the wall structure is highly deformed. The latter is also found for wires with $t=10 \mathrm{~nm}$ and $w=150 \mathrm{~nm}$. Hence, these samples are not investigated in detail and omitted within the paper.

[43] D. Stickler, R. Frömter, W. Li, A. Kobs, and H. P. Oepen, Integrated setup for the fabrication and measurement of magnetoresistive nanoconstrictions in ultrahigh vacuum, Rev. Sci. Instrum. 79, 103901 (2008).

[44] N. Mikuszeit, S. Pütter, R. Frömter, and H. P. Oepen, Magneto-optic Kerr effect: Incorporating the nonlinearities of the analyzer into static photometric ellipsometry analysis, J. Appl. Phys. 97, 103107 (2005).

[45] A. Berger, S. Knappmann, and H. P. Oepen, Magnetooptical Kerr effect study of ac susceptibilities in ultrathin cobalt films, J. Appl. Phys. 75, 5598 (1994).

[46] S. Mendach, J. Podbielski, J. Topp, W. Hansen, and D. Heitmann, Spin-wave confinement in rolled-up ferromagnetic tubes, Appl. Phys. Lett. 93, 262501 (2008).

[47] X. Liu, R. Sooryakumar, C. J. Gutierrez, and G. A. Prinz, Exchange stiffness and magnetic anisotropies in bcc $\mathrm{Fe}_{1-x} \mathrm{Co}_{x}$ alloys, J. Appl. Phys. 75, 7021 (1994).

[48] The exchange stiffness of $\mathrm{Fe}_{x} \mathrm{Co}_{100-x}$ alloys has been found to be almost independent of stoichiometry, exhibiting values of $30-35 \mathrm{pJ} / \mathrm{m}$ in the composition range of $32<x<70$. For a $\mathrm{Fe}_{18} \mathrm{Co}_{72} \mathrm{~B}_{10}$ alloy, a similar value $(A=29 \mathrm{pJ} / \mathrm{m})$ has been reported [49], while it has been demonstrated that varying the boron content has no impact on $A$ [50]. The same seems to hold for adding $\mathrm{Si}$ to $\mathrm{CoFe}$, as a similar exchange stiffness of $31.5 \mathrm{pJ} / \mathrm{m}$ is found for the Heusler compound $\mathrm{Co}_{2} \mathrm{FeSi}$ despite the huge amount of $\mathrm{Si}$ [51]. Hence, it is reasonable to assume a similar exchange stiffness for $\mathrm{Co}_{39} \mathrm{Fe}_{54} \mathrm{Si}_{7}$.

[49] C. Bilzer, T. Devolder, Joo-Von Kim, G. Counil, C. Chappert, S. Cardoso, and P. P. Freitas, Study of the dynamic magnetic properties of soft CoFeB films, J. Appl. Phys. 100, 053903 (2006). 
[50] T. Devolder, J. V. Kim, L. Nistor, R. Sousa, B. Rodmacq, and B. Dieny, Exchange stiffness in ultrathin perpendicularly magnetized $\mathrm{CoFeB}$ layers determined using the spectroscopy of electrically excited spin waves, J. Appl. Phys. 120, 183902 (2016).

[51] O. Gaier, J. Hamrle, S. Trudel, B. Hillebrands, H. Schneider, and G. Jakob, Exchange stiffness in the $\mathrm{Co}_{2} \mathrm{FeSi}$ Heusler compound, J. Phys. D 42, 232001 (2009).

[52] E. Feldtkeller, Ripple hysteresis in thin magnetic films, J. Appl. Phys. 34, 2646 (1963).

[53] H. B. Callen, R. L. Coren, and W. D. Doyle, Magnetization ripple and Arctic foxes, J. Appl. Phys. 36, 1064 (1965).

[54] K. J. Harte, Theory of magnetization ripple in ferromagnetic films, J. Appl. Phys. 39, 1503 (1968).

[55] O. Tchernyshyov and G.-W. Chern, Fractional Vortices and Composite Domain Walls in Flat Nanomagnets, Phys. Rev. Lett. 95, 197204 (2005).

[56] G.-W. Chern, H. Youk, and O. Tchernyshyov, Topological defects in flat nanomagnets: The magnetostatic limit, J. Appl. Phys. 99, 08Q505 (2006).

[57] P. M. Chaikin and T. C. Lubensky, Principles of Condensed Matter Physics (Cambridge University Press, Cambridge, England, 1995).
[58] A. Aharoni, Local demagnetization in a rectangular ferromagnetic prism, Phys. Status Solidi (b) 229, 1413 (2002).

[59] S. Pütter, N. Mikuszeit, E. Y. Vedmedenko, and H. P. Oepen, The effect of tilted edges on the shape anisotropy and stray field coupling of uniformly magnetized rectangular elements, J. Appl. Phys. 106, 043916 (2009).

[60] S Hankemeier, R. Frömter, N. Mikuszeit, D. Stickler, H. Stillrich, S. Pütter, E. Y. Vedmedenko, and H.P. Oepen, The Effect of Tilted Edges on the Shape Anisotropy and Stray Field Coupling of Uniformly Magnetized Rectangular Elements, Phys. Rev. Lett. 103, 147204 (2009).

[61] M. Redjdal, A. Kakay, M. Ruane, and F. Humphrey, Cross-tie walls in thin permalloy films, IEEE Trans. Magn. 38, 2471 (2002).

[62] N. Wiese, S. McVitie, J. N. Chapman, A. Capella-Kort, and F. Otto, On the scaling behaviour of cross-tie domain wall structures in patterned NiFe elements, Europhys. Lett. 80, 57003 (2007).

[63] A. Hubert and R. Schäfer, Magnetic Domains (SpringerVerlag, Berlin, 1998). 